\title{
Absorción de grasas termoxidadas. I. Reproducibilidad y exactitud de las técnicas analíticas previas a la evaluación de los lípidos no absorbidos.
}

\author{
Por G. Márquez-Ruiz, M. C. Pérez-Camino, V. Ruiz-Gutiérrez y M. C. Dobarganes. \\ Instituto de la Grasa y sus Derivados. C.S.I.C.- Avda. Padre Garcia Tejero n. $\stackrel{ }{4}$. \\ 41012 -SEVILLA (ESPAÑA).
}

\section{RESUMEN}

Absorción de grasas termoxidadas. I. Reproducibilidad y exactitud de las técnicas analíticas previas a la evaluación de los lípidos no absorbidos.

Se estudia la exactitud y reproducibilidad de las técnicas analíticas utilizadas en la extracción y separación de lipidos no absorbidos presentes en las heces de ratas Wistar alimentadas con dietas conteniendo aceites termoxidados.

Se ha encontrado una reproducibilidad muy elevada en la cuantificación de lípidos totales, ácidos grasos no polares y composición en ácidos grasos. Por otra parte, la comparación de los lípidos extraídos de las dietas con sus correspondientes aceites de partida demuestra en primer lugar una total recuperación de la grasa en el proceso de extracción y en segundo ausencia de alteración significativa durante el mismo.

Los lipidos no absorbidos se caracterizan por un elevado contenido en ácidos grasos polares y compuestos no saponificables.

PALABRAS-CLAVE: Aceite termoxidado - Dieta - Lipido no absorbido - Rata - Técnica analltica (exactitud) - Técnica anallitica (reproducibilidad)

\section{SUMMARY}

Absorption of thermoxidized fats. I. Reproducibility and accuracy of the previous analytical techniques used for the evaluation of non-absorbed lipids.

Reproducibility of the techniques used for isolation and separation of non-absorbed lipids present in feces from rats fed with diets containing heated oils are defined.

Highly reproducible results have been found for total lipids, nonpolar fatty acid compositions. Results obtaines from extracted diets demonstrate both the absence of significant alteration and the complete extraction of the fat when compared to those obtained directly from the oils.

Non-absorbed lipids from diets containing thermoxidized oils are characterized by high proportions of unsaponifiable compounds and polar fatty acids.

KEY-WORDS: Analytical technique (accuracy) - Analytical technique (reproducibility) - Diet - Non absorbed lipid - Rat - Thermoxidized oil.

\section{INTRODUCCION}

Entre las grasas consumidas después de haber sido sometidas a elevada temperatura, las utilizadas en el proceso de fritura son las que plantean una mayor controversia en lo que se refiere a sus propie- dades fisiológicas y nutricionales (1). Ello es debido, fundamentalmente, a que son ingeridas después de sufrir varios ciclos de calentamiento, durante los cuales se origina una creciente degradación de la grasa (2) (3) (4).

Aunque los estudios realizados con grasas de fritura demuestran la inexistencia de toxicidad en animales experimentales (5) (6) (7), la preocupación por limitar la alteración ha tenido como consecuencia el establecimiento de normas que, de forma indicativa o imperativa, sugieren el momento en que la grasa debe desecharse para consumo humano (8) (9) (10) (11).

La creciente utilización de la fritura como procedimiento clásico de preparación de alimentos y como nuevo proceso de fabricación de productos nuevos (12) (13) ha conducido, por otra parte, a la existencia de grandes cantidades de grasas de fritura que, una vez desechadas para consumo humano, son utilizadas en nutrición animal (14). Su uso como fuente de energía de bajo coste, lleva asociado la necesidad de profundizar en el conocimiento de su valor nutritivo tanto para conocer su rendimiento energético como para definir la influencia y el efecto de los compuestos de oxidación y polimerización presentes (15).

El objetivo global de estos estudios se resume en los siguientes puntos:

1) Establecer una metodología que permita cuantificar los principales grupos de compuestos de alteración tanto en las grasas ingeridas como en los lípidos no absorbidos.

2) Definir, a partir de una sistemática analítica reproducible, las tasas de absorción de los compuestos más característicos originados en la alteración termoxidativa, para deducir, en su caso, los que tienen mayor probabilidad de afectar negativamente el valor nutricional de la grasa.

3) Contribuir al establecimiento de bases objetivas que permiten relacionar los niveles de alteración con las propiedades fisiológicas de las grasas termoxidadas. 
En este primer trabajo se analiza la reproducibilidad y exactitud de las etapas previas a la cuantificación de los lípidos no absorbidos y se definen los principales grupos de compuestos presentes en los mismos como un paso necesario antes del planteamiento de los ensayos nutricionales.

\section{PARTE EXPERIMENTAL}

\subsection{Animales y dietas.}

Para la evaluación de las técnicas a utilizar en los posteriores ensayos nutricionales, se utilizaron dos grupos de cuatro ratas Wistar. Los animales fueron alimentados con dietas conteniendo un $12 \%$ de aceite de girasol fresco y calentado a $190^{\circ} \mathrm{C}$ durante 100 horas. La dieta alipídica purificada que sirve como base tiene la siguiente composición:

- HUMEDAD

$10,0 \%$

- PROTEINAS(caseina)

$19,8 \%$

- EXTRACTO LIBRE DE NITROGENO (glucosa+almidón).. $\quad 57,6 \%$

- CELULOSA ..................................................................... $5,4 \%$

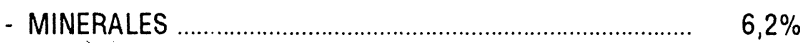

- COMPLEJO VITAMINICO _................................................ 1,0\%

El mantenimiento de los animales en jaulas individuales permitía cuantificar, cuando era necesario, la ingesta y las excreciones fecales y urinarias. Las heces eran recogidas diariamente y mantenidas en congelador hasta el momento de su desecación y extracción.

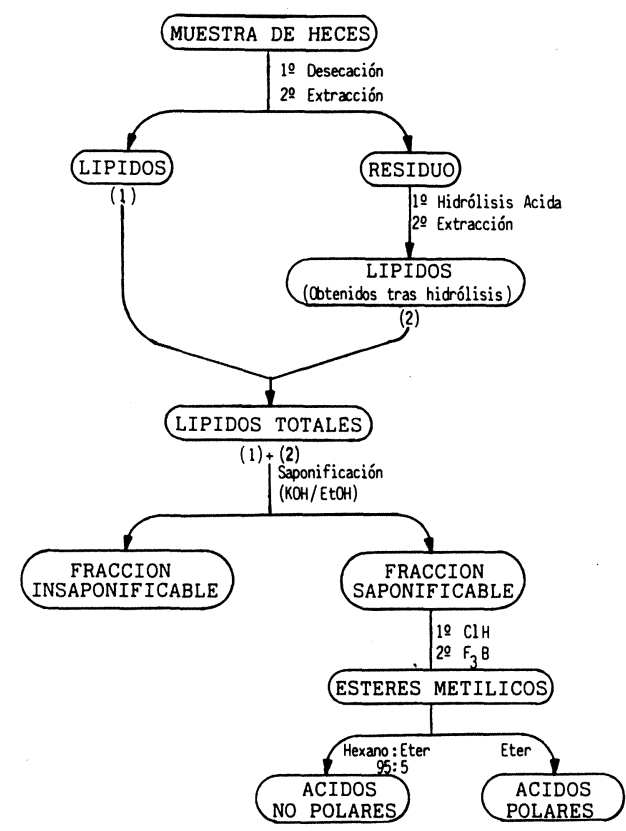

Figura 1

Esquema analítico general aplicado a los lípidos no absorbidos.

\subsection{Técnicas utilizadas.}

La fig. 1 resume el esquema general aplicado a la determinación de los lípidos no absorbidos presentes en las heces de los animales experimentales cuyas etapas se describen a continuación.

\subsubsection{Desecación}

Las heces obtenidas se colocan en cápsulas de fondo plano, previamente taradas, y se desecan en estufa de vacio a $50^{\circ} \mathrm{C}$ hasta peso constante.

\subsubsection{Extracción de los lípidos no absorbidos.}

La obtención de los lípidos se realiza en 2 etapas para garantizar su total extracción (16).

a) Obtención directa de los lípidos.

Las heces desecadas se pulverizan y se introducen en cartuchos de papel de filtro -unos $20 \mathrm{~g}$ de muestra por cada cartucho- recubierto de un tapón de algodón desengrasado. Los cartuchos se pasan a extractores Söxhlet, utilizando éter etílico como disolvente, y se mantienen en funcionamiento durante 7 horas, tiempo suficiente para garantizar la extracción total de la grasa. El extracto etéreo se somete a evaporación en un rotavapor bajo corriente de nitrógeno para eliminar el disolvente, hasta peso constante.

b) Obtención de lípidos previa hidrólisis ácida.

El residuo sólido de la operación anterior se somete a hidrólisis en frío con $400 \mathrm{ml}$ de $\mathrm{ClH} 3 \mathrm{~N}$ durante 2 horas. Se filtra sobre papel de filtro mojado, exento de materia grasa, al que se le agrega arena del mar purificada para facilitar el proceso de filtración, evitándose al mismo tiempo la pérdida de lípidos, y se lava el residuo con agua destilada hasta la desaparición de reacción ácida. A continuación se seca el doble papel de filtro conteniendo el residuo en estufa de vacío a $50^{\circ} \mathrm{C}$ hasta peso constante. Se introduce el doble filtro y el residuo seco en un cartucho de extracción y se extrae nuevamente con éter etílico por el procedimiento Söxhlet, durante 7 horas. El extracto etéreo se somete a evaporación con rotavapor bajo corriente de nitrógeno para eliminar el disolvente hasta peso constante.

Una vez extraídas y pesadas ambas fracciones de los lípidos presentes en heces, se toman cantidades proporcionales de las mismas para constituir la muestra representativa de los lípidos totales no absorbidos. ble.

\subsubsection{Determinación de la fracción insaponifica-}

Se ha seguido la NORMA UNE 55-004-73, partiendo de $1 \mathrm{~g}$ de grasa, dada la elevada cantidad de fracción insaponificable presente en las muestras. 


\subsubsection{Determinación de ácidos grasos totales.}

Las soluciones alcohólicas de jabones y las aguas de lavado, que quedan después de la separación del insaponificable, se reúnen en un embudo de decantación y se añade $\mathrm{CIH} 1 \mathrm{~N}$, hasta acidificación. Se deja enfriar, se extrae varias veces con porciones de 100 $\mathrm{ml}$ de éter etílico y se lavan las porciones etéreas reunidas con solución acuosa de $\mathrm{CINa}$ al $10 \%$. Por último, se añade $\mathrm{SO}_{4} \mathrm{Na}_{2}$ anhidro, se filtra sobre un matraz tarado y se evapora el disolvente en corriente de nitrógeno hasta peso constante.

\subsubsection{Determinación de ésteres metilicos.}

Obtención de los ésteres metílicos (17).

Se pesan 300-350 mg, con exactitud del $\mathrm{mg}$, de la muestra de ácidos grasos, en un matraz de $5 \mathrm{ml}$ al que se añaden $5 \mathrm{ml}$ de reactivo $F_{3} B 2 M$ en metanol. Se mantiene a ebullición durante 3 minutos, se interrumpe la calefacción y se agrega al matraz disolución acuosa saturada en $\mathrm{CINa}$, en cantidad suficiente para que al añadirle 3 ó $4 \mathrm{ml}$ de hexano, éste quede en el cuello del matraz. Se extrae repetidas veces con hexano para garantizar una buena extracción de los ésteres metílicos, evaporándose el disolvente en un rotavapor bajo corriente de nitrógeno.

Separación de los ésteres metílicos mediante cromatografía en columna de sílice.

Se sigue el método puesto a punto por Dobarganes et al. (18) con las siguientes modificaciones.

- Se utiliza una columna de vidrio de $40 \mathrm{~cm}$ de altura y 0,9 de d.i.

- Para la preparación de la columna cromatográfica se pesan $6 \mathrm{~g}$ de sílice y se añaden $20 \mathrm{ml}$ de hexano-éter etílico 95:5.

- Se pesan $300 \mathrm{mg}$ de ésteres metílicos, con exactitud del $\mathrm{mg}$, se disuelven en 5-8 $\mathrm{ml}$ de hexano-éter etílico 95:5 y, a continuación se transfiere a la columna cromatográfica.

- La fracción no polar se eluye con $50 \mathrm{ml}$ de hexanoéter etílico 95:5 y la elución de la fracción polar se realiza con $50 \mathrm{ml}$ de éter etílico y $50 \mathrm{ml}$ de cloroformo-metanol 2:1.

\subsection{Muestras utilizadas.}

\subsubsection{Evaluación de las etapas de desecación} y extracción.

La reproducibilidad de ambas etapas conjuntamente se ha obtenido a partir de 4 muestras de composiciones muy diferentes:

1.- Heces de animales alimentados con dieta que contiene $12 \%$ de aceite de girasol no calentado.
2.- Heces de animales alimentados con dieta que contiene $12 \%$ de aceite de girasol calentado 100 horas a $190^{\circ} \mathrm{C}$.

3.- Dieta conteniendo $12 \%$ de aceite de girasol no calentado.

4.- Dieta conteniendo $12 \%$ de aceite de girasol calentado durante 100 horas a $190^{\circ} \mathrm{C}$

Duplicados de $30 \mathrm{~g}$ de muestra son desecadas $y$, una vez exentas de humedad, extraidas.

La reproducibilidad y exactitud de las etapas se establece a partir de la cantidad de grasa obtenida y del porcentaje de ácidos grasos no alterados, realizado también por duplicado ( 8 datos por muestra).

2.3.2. Evaluación de las características generales de los lípidos no absorbidos.

Se han utilizado las siguientes muestras:

a) Lípidos no absorbidos de origen endógeno, procedentes de las heces de animales alimentados con dieta exenta de grasa.

b) Lípidos no absorbidos, tanto de la fracción obtenida por extracción directa como tras hidrólisis ácida, procedentes de animales alimentados con dietas conteniendo $12 \%$ de los aceites de girasol no calentado y termoxidado a $190^{\circ} \mathrm{C}$ durante 100 horas.

\section{RESULTADOS Y DISCUSION.}

Las Tablas I y || recogen los valores de lípidos totales y ácidos grasos no polares obtenidos para cada una de las cuatro muestras utilizadas para el análisis de la reproducibilidad y exactitud de las técnicas de desecación y extracción. Los resultados están expresados en porcentaje de grasa sobre muestra iniciai y porcentajes de ésteres metílicos no polares sobre lípidos totales, respectivamente.

Se ha partido, en el caso de las heces, de una única muestra homogénea $y$, por tanto, los resultados no incluyen las posibles diferencias existentes entre animales sino exclusivamente entre las variaciones debidas a las técnicas utilizadas. Los resultados de ambas Tablas indican una buena reproducibilidad ya que los límites de confianza de las medias, asumiendo una distribución de Student, son inferiores al $5 \%$, a pesar del elevado valor de $t$ para 3 grados de libertad $(t=3,182)$.

Aunque en los lípidos de heces sólo es posible analizar la reproducibilidad, las muestras que corresponden a la dieta a la que se ha añadido el aceite de composición conocida, son de gran interés para evaluar la exactitud de la determinación y la posibilidad de que existan modificaciones de la grasa durante las etapas analizadas. Como puede observar- 
Tabla I

Reproducibilidad y exactitud de las etapas de desecación y extracción ( $\mathrm{g} / 100 \mathrm{~g}$ muestra inicial)

\begin{tabular}{|c|c|c|c|c|}
\hline & $\begin{array}{l}\text { LIPIDOS HECES } \\
\text { (Dieta girasol no } \\
\text { calentado } 12 \% \text { ) }\end{array}$ & $\begin{array}{l}\text { LIPIDOS HECES } \\
\text { (Dieta girasol } \\
\text { termoxidado 12\%) }\end{array}$ & $\begin{array}{l}\text { DIETA } 12 \% \\
\text { Girasol no calentado }\end{array}$ & $\begin{array}{l}\text { DIETA } 12 \% \\
\text { Girasol termoxidado }\end{array}$ \\
\hline & 1,97 & 5,32 & 11,82 & 11,93 \\
\hline & 1,84 & 5,16 & 11,95 & 11,75 \\
\hline & 1,91 & 4,97 & 12,17 & 11,88 \\
\hline & 2,03 & 5,09 & 12,04 & 11,73 \\
\hline $\bar{x}$ & 1,94 & 5,13 & 11,99 & 11,84 \\
\hline$t s_{\bar{x}}$ & 0,13 & 0,22 & 0,15 & 0,19 \\
\hline
\end{tabular}

Tabla II

Reproducibilidad y exactitud del porcentaje de ácidos grasos no polares (g/100 g de lípidos)

\begin{tabular}{|c|c|c|c|c|}
\hline & $\begin{array}{l}\text { LIPIDOS HECES } \\
\text { (Dieta girasol no } \\
\text { calentado } 12 \%)\end{array}$ & $\begin{array}{l}\text { LIPIDOS HECES } \\
\text { (Dieta girasol } \\
\text { termoxidado } 12 \% \text { ) }\end{array}$ & $\begin{array}{l}\text { DIETA } 12 \% \\
\text { Girasol no calentado }\end{array}$ & $\begin{array}{l}\text { DIETA } 12 \% \\
\text { Girasol termoxidado }\end{array}$ \\
\hline & 50,92 & 29,58 & 97,32 & 57,41 \\
\hline & 51,13 & 28,34 & 98,12 & 57,92 \\
\hline & 51,84 & 29,12 & 97,62 & 56,83 \\
\hline & 51,73 & 29,31 & 98,01 & 56,12 \\
\hline & 52,64 & 28,83 & 97,31 & 56,34 \\
\hline & 51,28 & 30,12 & 96,82 & 57,12 \\
\hline & 50,41 & 29,11 & 97,34 & 55,88 \\
\hline & 51,63 & 29,84 & 96,12 & 54,45 \\
\hline $\bar{x}$ & 51,45 & 29,28 & 97,33 & 56,51 \\
\hline$t s_{\bar{x}}$ & 0,57 & 0,47 & 0,54 & 0,90 \\
\hline
\end{tabular}

se, la cantidad de grasa obtenida en ambos casos tiene una desviación extremadamente pequeña con respecto a la cantidad adicionada ( $12 \mathrm{~g}$ de aceite/88 $\mathrm{g}$ de dieta exenta de grasa), lo que indica al mismo tiempo la exactitud de la determinación y la extracción completa de la grasa.

En relación con la posibilidad de modificaciones durante la desecación y extracción, se han obtenido los ácidos no polares partiendo directamente de los aceites, encontrándose porcentajes de 97,5 y 55,2 para el aceite no calentado y termoxidado respectivamente. Como puede observarse en la Tabla II los valores obtenidos a partir de la dieta son muy similares mientras que se habrían obtenido valores significativamente menores en caso de alteración de la grasa.

Igualmente, en la Tabla III se encuentra la composición en ácidos grasos del aceite de girasol no calentado antes y después de ser sometido a las etapas de desecación y extracción de la dieta que lo contiene. Teniendo en cuenta que los errores de la determinación de los ácidos grasos pueden ser hasta del $5 \%$ para los ácidos que se encuentran en la muestra en proporción superior al $10 \%$ (19), puede concluirse que la alteración de la muestra no es significativa. 
Tabla III

Aceite de girasol no calentado y muestras sometidas a etapas previas: composición en ácidos grasos mayoritarios.

\begin{tabular}{|l|c|c|c|c|c|c|c|c|c|}
\hline \multirow{2}{*}{$\begin{array}{l}\text { ACIDOS } \\
\text { GRASOS }\end{array}$} & \multirow{2}{*}{$\begin{array}{c}\text { ACEITE } \\
\text { DE } \\
\text { GIRASOL }\end{array}$} & \multicolumn{4}{|c|}{ DESECACION 1 } & \multicolumn{4}{|c|}{ DESECACION 2 } \\
\cline { 2 - 9 } & \multicolumn{2}{|c|}{ EXTRACCION } & \multicolumn{2}{|c|}{ EXTRACCION 2 } & \multicolumn{2}{|c|}{ EXTRACCION 1 } & \multicolumn{2}{|c|}{ EXTRACCION 2 } \\
\cline { 2 - 9 }$C_{16: 0}$ & 8,2 & 8,8 & 9,1 & 8,3 & 8,1 & 9,1 & 8,0 & 9,0 & 9,1 \\
$C_{18: 0}$ & 5,0 & 5,4 & 4,9 & 4,7 & 4,6 & 5,5 & 4,7 & 5,1 & 4,9 \\
$C_{18: 1}$ & 27,8 & 27,5 & 26,8 & 27,3 & 26,7 & 29,2 & 28,9 & 26,7 & 28,1 \\
$C_{18: 2}$ & 59,0 & 58,3 & 59,2 & 59,7 & 60,6 & 56,2 & 58,4 & 59,2 & 57,9 \\
\hline
\end{tabular}

Tabla IV

Características generales de los lípidos no absorbidos.

\begin{tabular}{|l|l|c|c|c|c|}
\hline $\begin{array}{l}\text { LIPIDOS NO } \\
\text { ABSORBIDOS }\end{array}$ & $\begin{array}{l}\text { LIPIDOS* } \\
\text { TOTALES }\end{array}$ & $\begin{array}{c}\text { \% FRACCION } \\
\text { INSAPONIFICABLE }\end{array}$ & $\begin{array}{c}\% \text { ACIDOS NO } \\
\text { POLARES }\end{array}$ & $\begin{array}{c}\% \text { ACIDOS } \\
\text { POLARES }\end{array}$ & $\begin{array}{c}\text { ACIDEZ } \\
(\% \text { ol eico) }\end{array}$ \\
\hline $\begin{array}{l}\text { EXTRACCION DIRECTA } \\
\text { (girasol no calentado }\end{array}$ & 8,2 & 28,2 & 46,8 & 25,0 & 37,2 \\
$\begin{array}{l}\text { EXTRACCION DIRECTA } \\
\text { (girasol termoxidado) }\end{array}$ & 30,1 & 8,1 & 26,7 & 65,1 & 28,4 \\
$\begin{array}{l}\text { EXT. TRAS HIDRQ ISIS } \\
\text { (girasol no calentado) }\end{array}$ & 1,4 & - & 38,2 & 61,8 & - \\
$\begin{array}{l}\text { EXT. TRAS HIDRO ISIS } \\
\text { (girasol termoxidado) }\end{array}$ & 3,9 & - & 8,3 & 91,7 & - \\
\hline $\begin{array}{l}\text { ENDOGENOS } \\
\text { (dieta desengrasada) }\end{array}$ & $0,35 * \star$ & 29,2 & 52,1 & 18,7 & - \\
\hline
\end{tabular}

* \% sobre grasa ingerida

** \% sobre dieta ingerida

La Tabla IV resume los resultados obtenidos para las determinaciones realizadas sobre las 5 muestras utilizadas para conocer la composición de los lípidos no absorbidos.

En primer lugar, es importante comentar que las cantidades totales de lípidos neutros no absorbidos por $100 \mathrm{~g}$ de grasa ingerida son muy superiores en los animales alimentados con grasa alterada $(30,1 \mathrm{~g})$ frente a los alimentados con grasa no calentada $(8,2$ g).

En el análisis efectuado sobre la grasa extraída antes de la hidrólisis se encuentra un elevado valor para la fracción insaponificable $(28,2 \%)$ y para los ácidos grasos alterados $(46,8 \%)$ cuando la dieta incluye la grasa no calentada mientras que, en contraste, la dieta con grasa alterada se caracteriza por un alto porcentaje de ácidos grasos alterados $(65,1 \%)$ y cifras mucho menores de fracción insaponificable $(8,1 \%)$ y de ácidos grasos no alterados $(26,7 \%)$.

Además, el valor de la acidez libre de ambas muestras, incluida en la última columna, indica claramente la existencia de una cantidad elevada de ácidos esterificados cuando la dieta contiene el aceite termoxidado. Este hecho podría justificar la elevada cantidad de grasa no absorbida ya que, como es sabido, previo a la absorción es necesaria la hidrólisis 
enzimática por la lipasa pancreática, cuya acción es menos efectiva frente a moléculas complejas de triglicérido (20).

Por otra parte, las cantidades de lípidos obtenidos después de la hidrólisis ácida son bajas en relación con los lípidos neutros y destaca la práctica ausencia de fracción insaponificable. Sin embargo, el resultado de mayor importancia es que, en el caso de las grasas termoxidadas, esta fracción está mayoritariamente constituida por productos de alteración, lo que hace que su cuantificación sea imprescindible si se pretende determinar la cantidad de compuestos de degradación no absorbidos.

Finalmente, el análisis de los lípidos de origen endógeno demuestra la existencia de cantidades totales significativas $(0,35 \mathrm{~g}$ lípidos $/ 100 \mathrm{~g}$ de dieta base desengrasada ingerida) y de una importante contribución de la fracción insaponificable.

En resumen, los resultados obtenidos en este estudio permiten deducir las siguientes conclusiones:

1.- Junto a una buena reproducibilidad, las técnicas previas utilizadas para el aislamiento y separación de los lípidos no absorbidos garantizan una completa extracción de la grasa y la inexistencia de alteraciones significativas.

2.- Debido a las características químicas de las heces, la extracción directa de la grasa con disolventes no es suficiente para recuperar los lípidos no absorbidos. Es necesario realizar una segunda extracción después de hidrólisis ácida de la muestra, comprobándose que los compuestos obtenidos son, mayoritariamente, ácidos polares.

3.- Los lípidos no absorbidos, en general, se caracterizan por un elevado contenido en fracción insaponificable, cuya eliminación es necesaria antes de la cuantificación de los compuestos presentes en la fracción saponificable.

\section{AGRADECIMIENTO}

A la Junta de Andalucía y a la Comisión Interministerial de Ciencia y Tecnologia, por la financiación aportada. A la Sra. Mercedes Giménez, por la ayuda prestada.

\section{BIBLIOGRAFIA}

1. Artman, N. R.- "The chemical and biological properties of heated and oxidized fats".- Advances in Lipid Research 7 (1969) 245 330

2. Pokorny, J.- "Flavor chemistry of deep fat frying in oil". En "Flavor chemistry of lipid foods".- Ed. Min. D. B. y Smouse T. H. American Oil Chemists' Society E.E.U.U. (1989) 113-155.

3. Lumley, I. D.- "Polar compounds in heated oils". En "Frying of Food. Principles, changes, new approaches".- Varela, G., Bender, A. E. y Morton, I. D. (Ed.).- Ellis Horwood Ltd, Chichester Inglaterra (1988) 166-173.

4. Dobarganes, M. C., Pérez-Camino, M.C. y Márquez-Ruiz, G."Determinación de compuestos polares en aceites y grasas de fritura".- Grasas y Aceites 40 (1989) 35-38.

5. Billek, G.- "Heated fats in the diet". En "The role of fats in human nutrition".- Ed. Padley, F. B. y Podmone, I.- Ellis Horwood Chichester, Inglaterra (1985) 163-171.

6. Nolen, G. A., Alexander, J. C. y Artman, N. R.- "Long-term rat feeding study with used frying fats".- J. Nutr. 93 (1967) 337-348.

7. Lang, K. - "Die physiologischen wirkunger erhitzter Fette, insbesondere der Fritterfette". - Fette Seifen Anstrichmittel 75 (1973) $73-76$

8. Stevenson, S. G., Vaisewy-Genser, M. y Eskin, N.A.M.- "Quality control in the use of deep-frying oils".- J Am. Oil Chemists' Soc. 61 (1984) 1102-1108.

9. Castang, J.- "Etude sur les huiles de friture. Caractéres analy tiques et project de reglamentation".- Ann. Fals. Exp. Chim. 74 (1981) $701-718$

10. Meyer, H.- "Eine neue und einfache Schnellmethode zur Enfassung des oxidativen Zersetzungsgrade thermisch balastete Fette".- Fette Seifen Anstrichmittel 81 (1979) 524-533.

11. Boletín Oficial del Estado N. ${ }^{0}$ 26. 31 de Enero de 1989

12. Hauman, B. F.- "Trends in frying fat usage".- J. Am. Oil Chernists' Soc. 64 (1987) 789-805

13. Orthoefer, F. T.- "Oil use in the food service industry".- J. Am. Oil Chemists' Soc. 64 (1987) 795-799.

14. Steverink, A. T. G., De Vries, A. W. y Janssen, W. M. M. A."Influence of the composition of fat in poultry diets on the flavour of poultry mear". En "Strategies in food quality assurance: analy tical, industrial and legal aspects".- Ed. Baltes W. De Sikkel, Bélgica (1985) II 108-114

15. Andersen, J. O. Eggum, B.O. y Jacobsen, I.- "The significance of products of lipid oxidation upon the nutritive value of food".Lipid forum (1985) 148-153.

16. Toullec, R., Flauncy, J. y Rigaud, J.- "Estimation of lipids in feces. Separate extraction, amount and fatty acid composition of unsaponified lipids and insoluble complexes".- Ann. Biol. Anim. Bioch. Biophys 8 (1968) 281-289.

17. Metcalfe, L. D. y Schmitz, A. A.- "The rapid preparation of fatty acid esters for gas chromatographic analysis".- Anal. Chem. 33 (1961) 363-364.

18. Dobarganes, M. C., Pérez-Camino, M. C. y Gutiérrez GonzalezQuijano, R.- "Métodos analíticos de aplicación en grasas calentadas. I. Determinación de ésteres metllicos no alterados".Grasas y Aceites 35 (1984) 172-177.

19. Ackman, R. G.- "The analysis of fatty acids and material by gasliquid chromatography".- Progress in Chemistry of fats and other Lipids 12 (1972) 165-284.

20. Márquez-Ruiz, G., Pérez-Camino, M. C. y Dobarganes, M. C."In vitro action of pancreatic lipase on complex glycerides from thermally oxidized oils".- Fat Sci. Technol. (En revisión)

(Recibido: Mayo 1990) 